\title{
An application of a three-state all-or-none model to human eyelid conditioning data'
}

\author{
WILLIAM F, PROKASY \\ THE PENNSYLVANIA STATE UNIVERSITY
}

\begin{abstract}
The Bower-Theios three-state model was applied to eight sets of human eyelid conditioning data. In all instances, the prediction of stationarity in the intermediate state was not sustained. Earlier support of the model was concluded to have resulted from a bias in the method of testing for stationarity. Problems in the use of human conditioning data to test the model were discussed.
\end{abstract}

\section{Introduction}

Bower \& Theios (1964) show that a three-state, two-element pattern model provides an adequate description of some data from human eyelid conditioning. The model describes learning as a change from an initial state with $\mathrm{P}$ (response probability) $=\mathrm{O}$ to an intermediate state with $\mathrm{P}=\mathrm{k}$, a constant during that state, to the absorbing state where $p=1$. Successive trials in the intermediate state, which is the concern of this paper, constitute a sequence of Bernoulli trials to which all binomial statistics can be applied. Although its entire length cannot be determined from a sequence of trials, all trials separating the first CR from the last failure to make a $\mathrm{CR}$ before $\mathrm{P}$ becomes unity are included in the intermediate state. Trial-by-trial Ps separating the first $\mathrm{CR}$ and the last failure to make a $\mathrm{CR}$ will be referred to as the "intermediate state function," or as the "ISF."

Bower \& Theios (1964) obtained an ISF by lining up the data of all Ss who met criterion at the trial which followed the first CR. P, in blocks of three trials, was plotted following the first CR, with individual Ss dropped from the ISF as they met the criterion for complete learning. The ISF exhibited stationarity: i.e., $\mathrm{P}$ remained constant over trial blocks.

As noted elsewhere (Suppes \& Ginsberg, 1963; Prokasy, 1964) an ISF obtained with a decreasing $\mathrm{N}$ over trials may exhibit stationarity when, in fact, there are increments in $\mathbf{P}$. In this paper, ISFs from human eyelid conditioning are presented in which this bias does not exist.

\section{Procedure}

Eight sets of data were employed. Set A was obtained from I. Gormezano, and is composed of data taken from several studies with Ss given the same treatment combinations. Set B was obtained from W. Runquist ${ }^{2}$. Sets $C$ and $D$ were obtained from Prokasy (in press). Sets E through $\mathrm{H}$ were gathered with C. Allen in the Penn State laboratory. Sets G and $\mathrm{H}$ were obtained with $\mathrm{Ss}$ run under the masking procedure described by Spence, Homzie, \& Rutledge (1964).
ISFs were obtained only from those Ss in each set who met a learning criterion of 10 consecutive trials and who had at least 20 trials separating the first CR and criterion. Thus, ISFs of 20 trials in length were employed, with the same Ss contributing to each point. Both forward and backward ISFs were obtained from each set of data. The forward ISF had as its initial trial the trial which followed the first CR, while the backward ISF had as its terminal trial the trial which preceded the last failure to make a CR before criterion. Due to a limitation imposed by the number of trials, the ISF was only 12 trials long for set A.

\section{Results}

Forward and backward ISFs are presenred in Table I, together with information descriptive of the studies from which the data were obtained. The significant value of $x^{2}$ in all sets of data shows, by a median test, that the backward ISF is higher than the forward ISF. Moreover, increases in $\mathrm{P}$ over trials were observed in a number of instances, as indicated by the significant rank-order correlations between probability and ordinal-trial position. In neither instance, according to the model, should the null-hypothesis be rejected; hence, no set of data supports the model.

\section{Discussion}

It is unlikely that the discrepancy between these results and those reported by Bower \& Theios can be attributed solely to experimental conditions peculiar to these data. First, data set A came from the sample which Bower \& Theios used to support the model originally. Second, in only two sets (E \& F) is it possible to reject the hypothesis of stationarity when the ISF is plotted as a forward, decreasing-N function. Thus, $P$ increases during the intermediate state, but the forward, decreasing-N ISF is relatively insensitive to these increases. It can be concluded that the BowerTheios model does not provide an adequate description of human eyelid conditioning data.

This failure of the model should not be surprising in view of the fact that its boundary conditions were not met. The model can be applied appropriately only to data with an intial state $P$ of zero and a terminal state $P$ of unity. With the original data employed by Bower \& Theios it was not possible to obtain reliable estimates of initial state Ps, while the median $\mathrm{P}$ following the criterion run in the subset of those data constituting present Set A was .91. A similar result obtained with the remaining sets, the medians varying from .67 to .93. Moreover, mean initial state Ps in Sets E through $\mathrm{H}$ were found to vary from .10 to .25 . 
Table I

Forward and Backward ISFs from Eight Sets of Human Eyelid Conditioning Data ${ }^{\text {a }}$

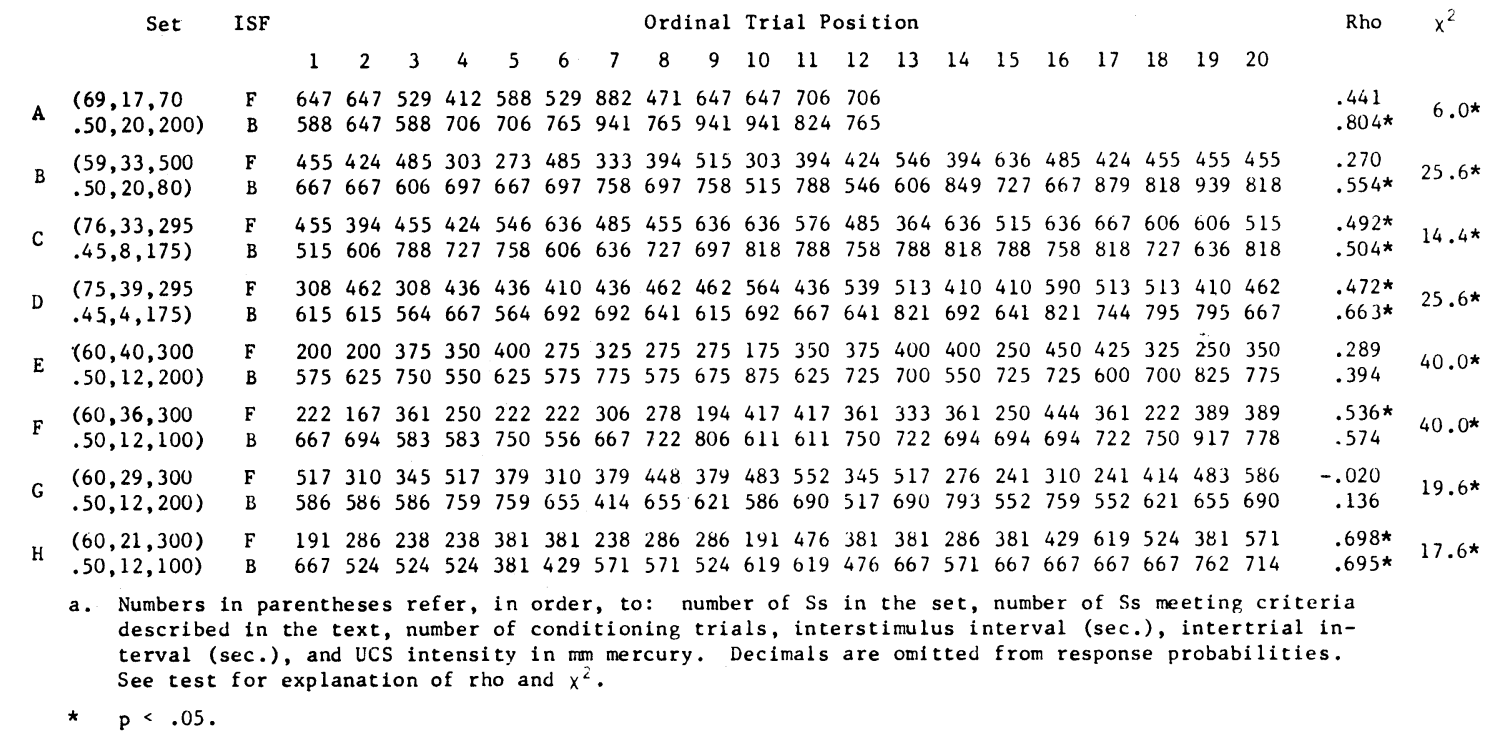

Recognizing these limitations, Theios and Brelsford (Brelsford, 1965; Theios \& Brelsford, 1965a, b) have developed a Markov model in which the initial and terminal state Ps do not have to be, respectively, zero and unity. While the newer model may be able to account for human eyelid conditioning, the data presently available do not provide a satisfactory vehicle for testing it. First, we do not have any data with reliable initial and terminal state Ps for individual Ss. Second, in those studies where reliable estimates of terminal state Ps are possible (e.g., Prokasy, in press), there are extensive individual differences. Finally, the latency distributions of individual Ss are frequently incomplete; i.e., the distribution of latencies is curtailed by UCS occurrence (Prokasy, in press). This necessarily results in an underestimate of S's response probability. It should be noted, too, that these distributions shift forward during conditioning, so that rises in $\mathrm{P}$ during the intermediate state are, at least in part, an artifact of this shift. Together, these two facts complicate parameter estimation and tests involving, particularly, sequential statistics.

\section{References}

Bower, G. H., \& Theios, J. A learning model for discrete performance levels. In R. C. Atkinson (Ed.), Studies in mathematical psychology. Stanford: Stanford University Press, 1964.
Brelsford, J. W., Jr. Experimental manipulation of state occupancy in a Markov model for avoidance conditioning. Report No. 7, Conditioning Research Laboratory, University of Texas, 1965.

Prokasy, W. F. Stimulus fluctuation, reactive inhibition and time between trials in classical eyelid conditioning. J. exp. Psychol., in press.

Prokasy, W. F. Pattern models, sampling theory, D $\left[\mathrm{A}\left(1-10^{-10}\right)\right] \ldots$ and some conditioning data. Paper read at Psychonomic Society Meeting, Niagara Falls, Ontarios, Canada, 1964.

Spence, K. W., Homzie, M. J., \& Rutledge, E. F. Extinction of the human eyelid $\mathrm{CR}$ as a function of the discriminability of the change from acquisition to extinction. J. exp. Psychol., 1964, $67,545-552$.

Suppes, P., \& Ginsberg, R. A fundamental property of all-or-none models, binomial distribution of responses prior to conditioning, with application to concept formation in children. Psychol. Rev., $1963,70,139-161$.

Theios, J., \& Brelsford, J. W., Jr. Eyelid conditioning in the rabbit as a Markov process. Paper read at the Midwestern Psychological Association Meeting, Chicago, Illinois, 1965a.

Theios, J., \& Brelsford, J. W., Jr. A Markov model for classical conditioning: application to eye-blink conditioning in rabbits. Report No. 9, Conditioning Research Laboratory, University of Texas, $1965 \mathrm{~b}$.

\section{Notes}

1. This research was supported by research grants G-18119 and GB-3875 from the National Science Foundation. Patricia Creveling and George Wolford aided in the running of subjects and collating of data. They were participants in the N.S.F. Undergraduate Research Participation Program, Grant GE-1057.

2. I would like to thank Professors I. Gormezano and W. Runquist for providing the original protocols from which data sets $\mathrm{A}$ and $\mathrm{B}$, respectively, were obtained. I would also like to thank Professor John Theios for some helpful comments on an earlier version of this paper. 